UB-ECM-PF 93/9

\title{
Thirring Model with Non-conserved Chiral Charge
}

\author{
D.Cabra $^{a, b}$, E.Moreno $^{c}$ and C.Naón ${ }^{a, b}$
}

\begin{abstract}
We study the Abelian Thirring Model when the fermionic fields have non-conserved chiral charge: $\Delta \mathcal{Q}_{5}=N$. One of the main features we find for this model is the dependence of the Virasoro central charge on both the Thirring coupling constant and $N$. We show how to evaluate correlation functions and in particular we compute the conformal dimensions for fermions and fermionic bilinears, which depend on the fermionic chiral charge. Finally we build primary fields with arbitrary conformal weight.
\end{abstract}

${ }^{a}$ Depto. de Física. Universidad Nacional de La Plata. CC 67, 1900 La Plata, Argentina.

${ }^{b}$ Consejo Nacional de Investigaciones Científicas y Técnicas, Argentina.

${ }^{c}$ Departament d'Estructura i Constituents de la Matèria, Universitat de Barcelona, Diagonal 647, 08028, Barcelona, Spain. 


\section{Introduction}

After the pioneering work of Belavin, Polyakov and Zamolodchikov [1], one of the most important steps towards the solution of 2D conformal field theories was given by Dotsenko and Fateev [2] (DF). These authors considered the Coulomb Gas Model with a non-trivial background charge at infinity. In this way they got a conformal anomaly as well as conformal weights that depend on this charge. With these basic ingredients they developed a systematic procedure to evaluate a general $n$-point correlation function of primary fields. Following their technique the operator algebra of non-trivial critical $2 \mathrm{D}$ systems such as the $q$-component Potts model [3] and $O(N)$ model were obtained [4].

The success of this approach led us to explore the possibility of implementing a similar idea but using a fermionic model as starting point. Thus, instead of the bosonic Coulomb gas system, in this paper we consider a modified version of the well-known Thirring model [5]. In order to introduce the analogous of DF's charge at infinity, we spoil the conservation of the chiral charge.

Our intention is to give an alternative formulation that could be useful in the identification of the discrete variables in a given statistical model with continuum fields.

A direct connection with statistical systems is lacking for an arbitrary Conformal Field Theory, except for some trivial cases such as the Ising Model, which is directly connected with a Majorana fermion via a Jordan Wigner transformation [6]. (The Ashkin-Teller [7] and Baxter [8] models have been also related to the usual Thirring model.)

This approach could facilitate the non-trivial task of relating lattice and field theoretical models via a Jordan-Wigner-like transformation.

In this paper we study the Abelian Thirring model in the case in which the chiral charge is not conserved. We found that the Virasoro central charge (VCC) of the resulting model depends on the "topological charge" and gives rise to a family of conformally invariant field theories with VCC given by:

$$
c=1+3 \frac{N^{2} \pi}{g^{2}}
$$

It is worthwhile noting the dependence of $c$ on the Thirring coupling constant, which appears as a purely topological effect. 
In the region in which the model is stable (which corresponds to $g^{2}>0$ ) this charge is greater than one, and hence the series (1.1) does not include for example Minimal Unitary models.

However one can study the region of negative couplings and in that case our model could be useful in the study of non-unitary statistical models like Scaling Lee-Yang model [10], etc.

Our model could be also relevant in the study of superconformal minimal models, for those models in the series for which the VCC is in the interval $[1,3 / 2]$ and other minimal unitary theories with additional symmetry, like $Z_{3}$ symmetry, Parafermionic theories, etc.

On the other hand, our formulation allows to obtain the primary fields in a simple way in contrast with the extremely complicated form that they take in the bosonic and fermionic formulation of coset models [11].

The paper is organized as follows: in Section II we present the model and show how to evaluate the VCC. Section III is devoted to the study of correlation functions and the evaluation of the conformal dimensions of the primary fields.

As a byproduct we obtain the conformal weights for some special bilinear fermionic operators. This allows us to obtain the field theoretical description of Baxter-like models.

\section{The Model}

Let us start with the euclidean Lagrangian:

$$
\mathcal{L}=-\bar{\Psi} i \not \partial \Psi-\frac{1}{2} g^{2}\left(\bar{\Psi} \gamma_{\mu} \Psi\right)^{2}
$$

which is invariant under the global abelian and abelian chiral groups:

$$
\begin{aligned}
& \Psi \rightarrow e^{i \alpha} \Psi \\
& \Psi \rightarrow e^{\gamma_{5} \beta} \Psi
\end{aligned}
$$

with $\alpha, \beta \in R$.

Our conventions for $\gamma$-matrices are:

$$
\begin{array}{r}
\left\{\gamma_{\mu}, \gamma_{\nu}\right\}=2 \delta_{\mu \nu} \quad, \quad \gamma_{5}=i \gamma_{0} \gamma_{1} \\
\gamma_{\mu} \gamma_{5}=i \epsilon_{\mu \nu} \gamma_{\nu} \quad, \quad \epsilon_{01}=1
\end{array}
$$


In the Path-Integral formulation of the theory we can eliminate quartic self interactions introducing an auxiliary field through the identity:

$$
\exp \left(\frac{1}{2} g^{2} \int d^{2} x\left(\bar{\Psi} \gamma_{\mu} \Psi\right)^{2}\right)=\int D A_{\mu} \exp \left(-\int d^{2} x\left(-g \bar{\Psi} \gamma_{\mu} \Psi A_{\mu}+\frac{1}{2} A_{\mu} A_{\mu}\right)\right)
$$

Then the partition function of the model is given by:

$$
\mathcal{Z}=\int D \bar{\Psi} D \Psi D A_{\mu} \exp \left(-\int d^{2} x\left(\bar{\Psi}(i \not \partial) \Psi+g \bar{\Psi} \gamma_{\mu} \Psi A_{\mu}-\frac{1}{2} A_{\mu} A_{\mu}\right)\right)
$$

In this form we can easily study the theory in a non-trivial chiral sector. In fact, the field $A_{\mu}$ is coupled minimally with the fermion current and has a natural interpretation as a gauge connection. Therefore a gauge configuration carrying a winding number $N$,

$$
\oint A_{\mu} d x^{\mu}=2 \pi N \text {. }
$$

will produce a break in the chiral symmetry. It is easy to show that the violation of the chiral charge is equal to $N$,

$$
\Delta \mathcal{Q}_{5}=N
$$

imposing the selection rule: the transition amplitudes between states are nonzero only if the variation of the chiral charge is equal to $N$.

In order to study the partition function (2.5) it is useful to decompose the auxiliary field as [12]:

$$
A_{\mu}=A_{\mu}^{(C l)}+a_{\mu}
$$

where $A_{\mu}^{(C l)}$ is a fixed background configuration which carries the topology and $a_{\mu}$ takes into account quantum fluctuations, i.e.

$$
D A_{\mu}=D a_{\mu} .
$$

With this choice the Lagrangian in eq.(2.5) takes the form:

$$
\mathcal{L}=\bar{\Psi} \not \not \partial \Psi+g \bar{\Psi} \gamma_{\mu} \Psi A_{\mu}^{(C l)}+g \bar{\Psi} \gamma_{\mu} \Psi a_{\mu}-\frac{1}{2} A_{\mu}^{(C l)} A_{\mu}^{(C l)}-a_{\mu} A_{\mu}^{(C l)}-\frac{1}{2} a_{\mu} a_{\mu} .
$$


Of course, by construction this partition function is independent of the background configuration $A_{\mu}^{(C l)}$ because any two configurations differ only in a shift in the quantum fluctuations.

We can decouple the quantum part of the auxiliary field $\left(a_{\mu}\right)$ from fermions making the following change of variables [13], [14]:

$$
\begin{aligned}
\Psi & =e^{i \eta+\gamma_{5} \phi} \chi \\
\bar{\Psi} & =\chi e^{-i \eta+\gamma_{5} \phi} \\
a_{\mu} & =-\frac{1}{g}\left(\epsilon_{\mu \nu} \partial_{\nu} \phi-\partial_{\mu} \eta\right)
\end{aligned}
$$

and choose the background configuration $A_{\mu}^{(C l)}$ satisfying:

$$
\partial_{\mu} A_{\mu}^{(C l)}=0
$$

Then the Lagrangian can be written as:

$$
\mathcal{L}=\bar{\chi}\left(i \not \partial+g A^{(C l)}\right) \chi+\frac{1}{g} \phi F^{(C l)}-\frac{1}{2 g^{2}}\left(\left(\partial_{\mu} \eta\right)^{2}+\left(\partial_{\mu} \phi\right)^{2}\right)-\frac{1}{2}\left(A_{\mu}^{(C l)}\right)^{2},
$$

where $F^{(C l)}=\epsilon_{\mu \nu} \partial_{\mu} A_{\nu}^{(C l)}$.

The jacobians associated with the change of variables (2.11) are given by:

$$
\begin{aligned}
& J_{f}=\exp \left\{-\frac{1}{2 \pi} \int d^{2} x\left(\left(\partial_{\mu} \phi\right)^{2}-2 g \phi F^{(C l)}\right)\right\} \\
& J_{b}=\operatorname{det}\left\{-\frac{1}{g^{2}}\left(\partial_{0}^{2}+\partial_{1}^{2}\right)\right\}
\end{aligned}
$$

and then the partition function can be written as:

$$
\mathcal{Z}=\operatorname{det}\left(-\frac{1}{g^{2}} \Delta\right) \times \mathcal{Z}_{\text {fermions }}\left[A^{(C l)}\right] \times \mathcal{Z}_{\eta-\text { boson }} \times \mathcal{Z}_{\phi-\text { boson }}\left[A^{(C l)}\right]
$$

where:

$$
\begin{gathered}
\mathcal{Z}_{\text {fermions }}\left[A^{(C l)}\right]=\int D \bar{\chi} D \chi \exp \left(-\int d^{2} x \bar{\chi}\left(i \not \partial+g A^{(C l)}\right) \chi\right) \\
\mathcal{Z}_{\eta-\text { boson }}=\int D \eta \exp \left(-\int d^{2} x \frac{1}{2 g^{2}}\left(\partial_{\mu} \eta\right)^{2}\right)
\end{gathered}
$$


and

$$
\mathcal{Z}_{\phi-\text { boson }}\left[A^{(C l)}\right]=\int D \phi \exp \left(-\int d^{2} x\left(\frac{1}{2 \pi}+\frac{1}{2 g^{2}}\right)\left(\partial_{\mu} \phi\right)^{2}-\left(\frac{1}{g^{2}}+\frac{1}{\pi}\right) \phi F^{(C l)}\right) .
$$

From the expression (2.15) for the partition function we can see that the fermionic and bosonic sectors are coupled only through the background configuration $A_{\mu}^{(C l)}$.

Now let us compute the conformal anomaly of this theory. Because of the independence of the partition function (2.18) on the particular election of the monopole background, we can choose it in such a way all the magnetic field be concentrated at infinity (the north pole of $S^{2}$, after compactification). With this election the three sectors, fermions, bosons and ghosts in the partition function (2.15) became separately conformal invariant on the complex plane $\mathcal{C}=S^{2}-\{$ north pole $\}$.

Consequently the Virasoro algebra of the whole theory is the sum of the three Virasoro algebras of the composing sub theories. We will see that, for the bosonic and the fermionic subsectors, the effect of the coupling with the background field is similar to the charge at infinity in the Dotsenko and Fateev Coulomb gas approach to conformal field theory Ref[2].

Let us start with the fermionic theory (2.16). Using the anomaly equation

$$
\partial_{\mu} j_{\mu}^{5}=\frac{1}{\pi} F^{(C l)}
$$

and the gauge condition (2.12) we can construct a pair of conserved currents [15]

$$
\begin{aligned}
& \mathbf{J}_{z}=\chi_{L}^{\dagger} \chi_{L}-\frac{1}{\pi} A_{z}^{(C l)} \\
& \mathbf{J}_{\bar{z}}=\chi_{R}^{\dagger} \chi_{R}+\frac{1}{\pi} A_{\bar{z}}^{(C l)} .
\end{aligned}
$$

The Noether charges associated with these currents takes the form, in radial quantization

$$
\begin{aligned}
\mathcal{Q}_{z} & =\oint d z \mathbf{J}_{z} \\
\mathcal{Q}_{\bar{z}} & =\oint d \bar{z} \mathbf{J}_{\bar{z}} .
\end{aligned}
$$

But using the monopole quantization condition (and the fact that the monopole has support at infinity) we see that the effect of the background field is to 
create a charge at infinity of value $N$. This charge changes the neutral charge condition of the holomorphic and anti-holomorphic vacuum expectation values and consequently there is a shift in the conformal dimension of the primary fields. At the level of the energy-momentum tensor, this shift is produced by the appearance of an extra term, which takes into account the changes in the conformal dimensions. The total $T_{z z}$ component of the energy-momentum tensor takes the form

$$
T=\left(\chi_{L}^{\dagger} \partial_{z} \chi_{L}-\partial_{z} \chi_{L}^{\dagger} \chi_{L}\right)+N \partial_{z}\left(\chi_{L}^{\dagger} \chi_{L}\right)
$$

and there is a similar equation for the $T_{\bar{z} \bar{z}}$ component. Using eq.(2.24) we can easily compute the Virasoro central charge of this fermionic theory and we find

$$
c_{\text {fermions }}=1-3 N^{2} .
$$

Now we can evaluate the contribution from the bosonic fields $\eta$ and $\phi$. For the $\eta$-field one get the well-known result:

$$
c_{\eta-\text { boson }}=1 .
$$

(This can be seen making a harmless rescaling of the $\eta$-field: $\tilde{\eta}=\frac{\sqrt{\pi}}{g} \eta$ ).

In order to evaluate the contribution coming from the $\phi$ field it is useful to make the following rescaling

$$
\tilde{\phi}=\sqrt{1+\frac{\pi}{g^{2}}} \phi,
$$

which leads the action to the form:

$$
S[\phi]=\frac{1}{2 \pi}\left(\int d^{2} x\left(\partial_{\mu} \tilde{\phi}\right)^{2}-Q \int d^{2} x \tilde{\phi} F^{(C l)}\right),
$$

where:

$$
Q=\sqrt{1+\frac{\pi}{g^{2}}} .
$$

The analysis of this theory is closely similar to the fermionic one. In particular it is easy to convince oneself that the coupling with the monopole produces a background charge at infinity of value $N Q$ (Ref.[16]). Then the modification that suffers the energy-momentum tensor is given by

$$
\Delta T=Q \partial_{z}^{2} \tilde{\phi}
$$


and the conformal anomaly takes the form

$$
c_{\phi-b o s o n}=1+3 N^{2}\left(1+\frac{\pi}{g^{2}}\right) .
$$

Finally we have to take into account the contribution coming from the Laplacian in equation (2.15). The determinant of the Laplacian operator can be exponentiated using anticommuting ghosts in the form:

$$
\operatorname{det}\left(-\frac{1}{g^{2}} \Delta\right)=\int D \bar{\eta} D \eta \exp \left(-\frac{1}{g^{2}} \int d^{2} x \bar{\eta} \Delta \eta\right)
$$

The corresponding conformal charge is:

$$
c_{\text {ghosts }}=-2 \text {. }
$$

At this point we can evaluate the total central charge of the model just by adding the four independent contributions eqs.(2.25),(2.26), (2.31), and (2.33) which yields to:

$$
c_{\text {total }}=1+3 \frac{\pi N^{2}}{g^{2}} .
$$

We see that the central charge of this model depends both on the coupling constant $g^{2}$ and on the topological charge $N$, this last being connected with the non-conservation of the chiral charge (eq.(2.7)).

It is worthwhile noting the resemblance of this result with the one obtained by Dotsenko and Fateev [2]. In this sense our result could be viewed as a fermionic version of DF's construction. Two important differences should be stressed, however. Firstly, we have a conformal charge $c>1$ whereas $\mathrm{DF}$ got $c<1$. As a consequence, the FQS series [17] is not described by our model, at least for values of the coupling constant $g^{2}$ which lead to a unitary theory. Finally, it is remarkable the dependence of the central charge on the coupling constant $g^{2}$, which arises as a topological effect. 


\section{Correlation Functions and Primary Fields}

Due to the presence of the monopole in the fermionic partition function eq.(2.19) only chirality violating operators have a non-zero vacuum expectation value. For a given fermionic operator $\mathcal{O}$ its vacuum expectation value is zero unless the selection rule

$$
\left[\mathcal{Q}_{5}, \mathcal{O}\right]=N \mathcal{O}
$$

holds, where $N$ is the chiral charge. It is easy to verify that the fermion bilinears $\bar{\Psi}_{R} \Psi_{R}$ are eigenfunctions of the chiral charge operator with eigenvalue 1, and the bilinears $\bar{\Psi}_{L} \Psi_{L}$ are eigenfunctions of the chiral charge operator with eigenvalue -1 . Then, for $N>0$, a fermionic operator with a non-trivial vacuum expectation value contains a number of bilinears $\bar{\Psi}_{R} \Psi_{R}$ which exceeds in $N$ the number of bilinears $\bar{\Psi}_{L} \Psi_{L}$ (for $N<0$ we change $R \leftrightarrow L$ ). After the decoupling change of variables eq.(2.11) the fermionic operator $\mathcal{O}$ will be accompanied by bosonic vertex operator

$$
V=e^{\int \mathbf{j}(x) \phi(x)}
$$

with

$$
\mathbf{j}(x)=\sum_{i} \alpha_{i} \delta\left(x-x_{i}\right) ; \quad \sum_{i} \alpha_{i}=-2 N .
$$

Now let analyze the bosonic partition function eq.(2.18). The vertex operator eq.(3.2) adds a new term to the action

$$
\Delta S_{b}=-\int \mathbf{j}(x) \phi(x) d^{2} x
$$

Then after the rescaling (2.26) the bosonic effective action reads

$$
S_{b}=\frac{1}{2 \pi} \int\left\{(\partial \phi)^{2}-\phi\left[Q F^{(C l)}+\frac{2 \pi}{Q} \mathbf{j}+\frac{2 \pi}{Q} \mu\right]\right\}
$$

where we included an external source $\mu$. ( $Q$ was defined in eq.(2.29)).

The path integral over the constant zero mode of the Laplacian operator imposes, as usual, the neutrality charge condition:

$$
\frac{1}{4 \pi} \int\left(Q F^{(C l)}+\frac{2 \pi}{Q} \mathbf{j}+\frac{2 \pi}{Q} \mu\right) d^{2} x=N\left(Q-\frac{1}{Q}\right)+\frac{1}{2 Q} \int \mu d^{2} x=0 .
$$


Then, in order to have a non-trivial result for the vacuum expectation value we need the presence of the additional source $\mu$ satisfying

$$
\int \mu d^{2} x=\left(Q^{2}-1\right) N=\frac{\pi}{g^{2}} .
$$

This contribution can be obtained in a natural way from the term

$$
e^{-\frac{1}{g^{2}} \int A_{\mu} l^{\mu}}
$$

which comes from a source term for the currents in the original Lagrangian (eq.(2.1)).

Then a non-trivial vacuum expectation has the form

$$
\begin{gathered}
<\mathcal{H}[\bar{\Psi}, \Psi] e^{\int j_{\mu} l^{\mu}}>=\int D \bar{\chi} D \chi D \phi \exp \left(\int \bar{\chi}\left(\not \partial \partial+g A^{(C l)}\right) \chi\right) \times \\
\exp \left(\frac{1}{2 \pi} \int\left\{(\partial \phi)^{2}-\phi\left[Q F^{C l}+\frac{2 \pi}{Q} \mathbf{j}-\frac{\pi}{g^{2} Q} \epsilon_{\mu \nu} \partial_{\mu} l_{\nu}\right]\right\}\right) \mathcal{H}[\bar{\chi}, \chi]
\end{gathered}
$$

where $\mathcal{H}[\bar{\chi}, \chi]$ is a fermionic composite operator satisfying the chiral selection rule eq.(3.1). The constraint eq.(3.7) is translated into the following condition for the source $l_{\mu}$

$$
\frac{1}{2 \pi} \int \epsilon_{\mu \nu} \partial_{\mu} l_{\nu} d^{2} x=4 \pi N,
$$

i.e., $l_{\mu}$ is a monopole of charge $N$.

Because of the freedom in the election of the classical background $A_{\mu}^{C l}$ in the total partition function eq.(2.15) we can choose $A_{\mu}^{C l}=l_{\mu}$. Finally integrating the remaining (non-zero modes) bosonic degrees of freedom we obtain the vacuum expectation value of $\mathcal{O}=\mathcal{H}[\bar{\Psi}, \Psi] e^{\int j_{\mu} l^{\mu}}$

$$
\begin{gathered}
<\mathcal{O}>=\int D \bar{\chi} \chi \exp \left(\int \bar{\chi}\left(i \not \partial+g A^{(C l)}\right) \chi\right) \mathcal{H}[\bar{\chi}, \chi] \times \\
\exp \left(-\frac{1}{8 \pi Q^{2}} \int\left(F^{C l}[l(x)]+2 \pi \mathbf{j}(x)\right) \Delta^{-1}(x, y)\left(F^{C l}[l(y)]+2 \pi \mathbf{j}(y) d^{2} x d^{2} y\right)\right),
\end{gathered}
$$

where $\Delta^{-1}(x, y)$ is the Green function of the Laplacian. 
The next task of this section is to construct the primary fields of the model and evaluate their conformal weights. As usual, given a conformal field $\phi_{h}$ we can evaluate its conformal dimension from the O.P.E with the energy-momentum tensor. Using the usual normalization in CFT this O.P.E. reads:

$$
T(z) \phi_{h}(w)=\frac{h}{(z-w)^{2}} \phi_{h}(w)+\frac{1}{z-w} \partial_{w} \phi_{h}(w)+r . t . .
$$

In the factored version of the model this can be achieved by evaluating the O.P.E's in each sector separately since our basic fields $\Psi$ can be expressed in terms of the fermion fields $\chi$, the bosonic fields $\phi$ (both coupled only to the background) and the free boson $\eta$ (see eq.(2.11)). (As usually happens in abelian theories the ghost sector appears completely decoupled).

We shall first consider the fermionic sector (2.16). The corresponding EMT was given in eq.(2.24) and for the O.P.E's with the fermionic fields $\chi_{R}$, $\chi_{L}, \bar{\chi}_{R}$ and $\bar{\chi}_{L}$ we get:

$$
\begin{aligned}
T_{\eta}(z) \chi_{R}(\bar{w})=\bar{T}_{\eta}(\bar{z}) \bar{\chi}_{R}(w)=T_{\eta}(z) \bar{\chi}_{L}(\bar{w})=\bar{T}_{\eta}(\bar{z}) \chi_{L}(w)=0, \\
\bar{T}_{\eta}(\bar{z}) \chi_{R}(\bar{w})=\frac{1-N}{2} \frac{1}{(\bar{z}-\bar{w})^{2}} \chi_{R}(\bar{w})+\frac{1}{(\bar{z}-\bar{w})} \partial_{\bar{w}} \chi_{R}(\bar{w}), \\
T_{\eta}(z) \bar{\chi}_{R}(w)=\frac{1-N}{2} \frac{1}{(z-w)^{2}} \bar{\chi}_{R}(w)+\frac{1}{(z-w)} \partial_{w} \bar{\chi}_{R}(w), \\
T_{\eta}(z) \chi_{L}(w)=\frac{1+N}{2} \frac{1}{(z-w)^{2}} \chi_{L}(w)+\frac{1}{(z-w)} \partial_{w} \chi_{L}(w), \\
\bar{T}_{\eta}(\bar{z}) \bar{\chi}_{L}(\bar{w})=\frac{1+N}{2} \frac{1}{(\bar{z}-\bar{w})^{2}} \bar{\chi}_{L}(\bar{w})+\frac{1}{(\bar{z}-\bar{w})} \partial_{\bar{w}} \bar{\chi}_{L}(\bar{w}) .
\end{aligned}
$$

Concerning the $\eta$-boson, we are interested in the conformal dimension of the vertex operator:

$$
\Phi_{\alpha}^{(\eta)}(z, \bar{z}) \equiv: e^{i \alpha \eta(z, \bar{z})}: .
$$

From the O.P.E. with the corresponding EMT we obtain:

$$
h=\bar{h}=\frac{g^{2}}{8 \pi} \alpha^{2}
$$


Finally we evaluate the O.P.E. of the $\phi$-vertex operator:

$$
\Phi_{\alpha}^{(\phi)}(z, \bar{z}) \equiv: e^{i \alpha \phi(z, \bar{z})}:,
$$

with the EMT given in eq.(2.30). The result is:

$$
\begin{aligned}
& T_{\phi}(z) \Phi_{\alpha}^{(\phi)}(w, \bar{w})=\frac{\alpha}{8}\left(2 N-\frac{\alpha}{1+\frac{\pi}{g^{2}}}\right) \frac{1}{(z-w)^{2}} \Phi_{\alpha}^{(\phi)}(w, \bar{w})+\ldots \\
& \bar{T}_{\phi}(\bar{z}) \Phi_{\alpha}^{(\phi)}(w, \bar{w})=\frac{\alpha}{8}\left(2 N-\frac{\alpha}{1+\frac{\pi}{g^{2}}}\right) \frac{1}{(\bar{z}-\bar{w})^{2}} \Phi_{\alpha}^{(\phi)}(w, \bar{w})+\ldots
\end{aligned}
$$

Now we can write down the conformal weights associated to the original fermions $\Psi_{R}, \Psi_{L}, \bar{\Psi}_{R}$ and $\bar{\Psi}_{L}$ just by adding the previous fermionic contributions (3.15), (3.16) and setting $\alpha=1$ in (3.18) and (3.20). Thus we obtain:

$$
\begin{aligned}
h_{\Psi_{R}} & =\frac{N}{4}-\frac{g^{2}}{8\left(g^{2}+\pi\right)}+\frac{g^{2}}{8 \pi}, \\
\bar{h}_{\Psi_{R}} & =\frac{N}{4}-\frac{g^{2}}{8\left(g^{2}+\pi\right)}+\frac{g^{2}}{8 \pi}+\frac{1-N}{2}, \\
h_{\bar{\Psi}_{R}} & =\frac{N}{4}-\frac{g^{2}}{8\left(g^{2}+\pi\right)}+\frac{g^{2}}{8 \pi}+\frac{1-N}{2}, \\
\bar{h}_{\bar{\Psi}_{R}} & =\frac{N}{4}-\frac{g^{2}}{8\left(g^{2}+\pi\right)}+\frac{g^{2}}{8 \pi} \\
h_{\Psi_{L}} & =-\frac{N}{4}-\frac{g^{2}}{8\left(g^{2}+\pi\right)}+\frac{g^{2}}{8 \pi}+\frac{1+N}{2}, \\
\bar{h}_{\Psi_{L}} & =-\frac{N}{4}-\frac{g^{2}}{8\left(g^{2}+\pi\right)}+\frac{g^{2}}{8 \pi}, \\
h_{\bar{\Psi}_{L}} & =-\frac{N}{4}-\frac{g^{2}}{8\left(g^{2}+\pi\right)}+\frac{g^{2}}{8 \pi}, \\
\bar{h}_{\bar{\Psi}_{L}} & =-\frac{N}{4}-\frac{g^{2}}{8\left(g^{2}+\pi\right)}+\frac{g^{2}}{8 \pi}+\frac{1+N}{2}
\end{aligned}
$$

It is interesting to observe that the scaling dimensions given by $\Delta=h+\bar{h}$ do not depend on the chiral charge $N$. On the contrary, the spins $S=h-\bar{h}$ are changed by the background. We have, for instance, 


$$
\begin{gathered}
\Delta_{\Psi_{R}}=\frac{1}{2}+\frac{g^{2}}{4 \pi}-\frac{g^{2}}{4 \pi\left(1+g^{2} / \pi\right)}, \\
S_{\Psi_{R}}=\frac{1-N}{2} .
\end{gathered}
$$

We also note that (3.23) exactly coincides with the result obtained in Ref.[21] where the fermionic correlator was computed in the $N=0$ sector.

We will now focus our attention on primary fields that are built as fermionic bilinears. These are the energy-density and crossover operators which are defined, respectively, as:

$$
\begin{gathered}
\varepsilon(z, \bar{z})=\bar{\Psi}_{R} \Psi_{R}(z, \bar{z})+\bar{\Psi}_{L} \Psi_{L}(z, \bar{z}), \\
C_{r}(z, \bar{z})=\bar{\Psi}_{R} \bar{\Psi}_{L}(z, \bar{z}), \quad \bar{C}_{r}(z, \bar{z})=\Psi_{R} \Psi_{L}(z, \bar{z}) .
\end{gathered}
$$

(Strictly speaking the crossover operator is defined, for $N=0$, as the sum $\mathcal{C}_{r}=C_{r}+\bar{C}_{r}$. However in the case at hand $N \neq 0$, eventhough $C_{r}$ and $\bar{C}_{r}$ have the same scaling dimensions, their spins are different and consequently they do not add to a primary field). The conformal behavior of this operators is of particular importance in view of the close connection between the Thirring model and some lattice spin systems like the Ashkin-Teller Ref.[7] and Baxter models Ref.[8]. These two systems can be roughly described as the superposition of two Ising lattices which interact through 4-spin couplings. In the context of the Ashkin-Teller model $\varepsilon(x)$ accounts for temperature fluctuations, whereas $C_{r}(x)$ describes the difference between the nearest-neighbor couplings of the two Ising variables Ref.[22].

Combining adequately the conformal dimensions of the constituents (fermions and vertex operators) we have:

$$
\begin{aligned}
h_{\varepsilon} & =\bar{h}_{\varepsilon}=\frac{1}{2}\left(\frac{1}{1+\frac{g^{2}}{\pi}}\right), \\
h_{C_{r}} & ==\bar{h}_{\bar{C}_{r}}=-\frac{N}{2}+\frac{1+\frac{g^{2}}{\pi}}{2}, \\
\bar{h}_{C_{r}} & ==h_{\bar{C}_{r}}=\frac{N}{2}+\frac{1+\frac{g^{2}}{\pi}}{2} .
\end{aligned}
$$


It must be stressed that the scaling dimensions for the energy and the crossover operators are related as:

$$
\Delta_{C_{r}}=\Delta_{\bar{C}_{r}}=1+\frac{g^{2}}{\pi}=\frac{1}{\Delta_{\varepsilon}}
$$

This relation had been conjectured in Ref.[23], [24] and was first derived in Ref.[25] for the $N=0$ case (see also Ref.[26] for a proof of this identity in the path-integral framework). Eq.(3.28) shows that this relation holds irrespective of the boundary conditions for the fermionic fields.

Since the theory at hand has $c>1$ we must be able to construct primary fields with arbitrary conformal weights. Apart from special cubic and quartic combinations of fermions, the unique way to obtain this result is to deal with non-local operators Ref.[27] like:

$$
\Phi_{\alpha}(z) \equiv: e^{i \alpha \int_{C_{z}} d w j(w)}:,
$$

where:

$$
j(z)=2 \pi: \bar{\Psi}_{R} \Psi_{L}:(z)=2 \pi: \bar{\chi}_{R} \chi_{L}:(z),
$$

with:

$$
j(z) j(w)=-\frac{1}{(z-w)^{2}}
$$

and $C_{z}$ is a complex path starting at zero and ending at $z$.

In order to calculate the O.P.E. of $\Phi_{\alpha}(w)$ with the EMT it is useful to express the last one in the Sugawara form:

$$
T(z)=-\frac{1}{2}: j(z) j(z):+\frac{i N}{2}: \partial_{z} j(z): .
$$

Using this expression for the EMT together with (3.31) we obtain:

$$
h=\frac{\alpha(\alpha-N)}{2} .
$$

\section{Conclusions}

It is clear from the results of this paper that the conformal properties of the Thirring model are modified when we consider a non-zero chiral charge sector. 
The conformal anomaly, equal to 1 in the zero charge sector, has an additional contribution proportional to the chiral charge an inversely proportional to the coupling constant

$$
c=1+3 \frac{\pi N^{2}}{g^{2}}
$$

This result is very similar to the conformal anomaly of the Dotsenko and Fateev's modified Coulomb gas but with an opposite sign. Here the ratio $\frac{N}{g^{2}}$ plays the role of the (imaginary) charge at infinity in DF's work. Of course such a modification implies a change in the whole conformal algebra, and in particular a new set of primary and descendants conformal fields. For the particular case of the fermions we found that only the spins are $N$-dependent, the scaling dimensions are independent of the chiral charge.

As it was shown by Luther and Peschel [9] one can generalize the JordanWigner transformation for the spin operators on a lattice model to the continuum case. This fact enables one to study the lattice Baxter model in terms of the continuum Thirring model (for the $N=0$ case). In this context the fermionic bilinears $\varepsilon(3.25)$ and $C_{r}(3.26)$ play a central role.

We have computed the conformal weights for these operators, when the fermion fields associated to the corresponding Jordan-Wigner transformations satisfy non-trivial boundary conditions. We found that $h_{\epsilon}$ and $\bar{h}_{\epsilon}$ are not affected by having $\mathrm{N}$ different from zero. On the other hand, the crossover operator does change in this new situation: it splits down into two primary operators with equal scaling dimensions but opposite spins. These results can be understood by recalling that the energy density is a rotationally invariant operator while the crossover is not.

The non-trivial conditions satisfied by the fermionic fields lead to nontrivial constraints between the spin variables in the related lattice model. (This can be seen by using the Jordan-Wigner transformation which relates the usual Thirring model with the Baxter model) It would be interesting to try to generate these constraints in the lattice by the addition of an interaction between the spins.

As mentioned in the Introduction, the central charge that we have obtained for a given "topological charge" $N$ can be matched with the VCC of certain Superconformal Minimal Models (those for which the VCC is in the interval $[3 / 2,1]$ ) by a suitable choice of the coupling $g^{2}$. For those cases it would be interesting to construct the superpartner of the EMT and study 
the connection with the realization of Superconformal invariance in related lattice models.

The addition of a mass term to the Lagrangian (2.1) corresponds to a perturbation with an operator of dimension $h=\bar{h}=1 / 2\left(1 / 1+g^{2} / \pi\right)$. In that case one can study the Renormalization Group flow between two adjacent conformal field theories by evaluating the Zamolodchikov's $c$-function [28].

\section{Acknowledgements}

E.F.M. thanks J. Soto and. J.I. Latorre for usefull comments. D.C.Cabra and C. Naón are partially supported by CONICET. E.F.M. acknowledges a PostDoctoral fellowship from the Ministerio de Educación y Ciencia. Partially supported by CICYT project AEN90-0033.

\section{References}

[1] A.A.Belavin, A.M.Polyakov and A.B.Zamolodchikov, Nucl.Phys. B241, 333, (1984).

[2] Vl.S.Dotsenko and V.A.Fateev, Nucl.Phys. B240, 312, (1984).

[3] Vl.S.Dotsenko, J. Stat. Phys. 34, 781, (1984); Nucl.Phys. B235[FS11], 74, (1984). F.W.Wu, Rev. Mod. Phys. 54, 235, (1982).

[4] B.Nienhuis, Phys.Rev.Lett 49, 1062, (1982).

[5] W.Thirring,Ann. Phys. 3, 91, (1958).;B.Klaiber, Lectures in Theoretical Physics, Boulder 1967, eds. Gordon and Breach, New York, 141, (1968).

[6] T.Schultz, D.Mattis and E.Lieb, Rev. Mod. Phys. 36, 856, (1964).

[7] J.Ashkin and E.Teller, Phys.Rev. 64, 178, (1943).

[8] R.Baxter, Phys.Rev.Lett. 26, 832, (1971).

[9] A.Luther and I.Peschel, Phys.Rev.B 12, 3908, (1975).

[10] M.E.Fischer, Phys.Rev.Lett. 40, 1610, (1978). 
[11] K.Gawedzki and A.Kupiainen, Phys. Lett. B215, 119, (1988).

[12] K.Bardacki and M.Crescimanno, Nucl.Phys. B313, 269, (1989).

[13] R.Roskies and F.A.Schaposnik, Phys.Rev.D 23, 558, (1981).

[14] R.E.Gamboa Saravi, F.A.Schaposnik and J.E.Solomin, Nucl.Phys. B181, 239, (1981).

[15] E.F.Moreno, Int.J.Mod.Phys.A 7, 2179, (1992).

[16] D.C.Cabra and E.F.Moreno, J. Phys. A: Math. Gen. 23, 4711, (1990).

[17] D.Friedan, Z.Qiu and S.Shenker, Phys. Rev. Lett. , 52, 1575, (1984).

[18] M.V.Manias, C.M.Naón and M.Trobo, Int.J.Mod.Phys.A 5, 2853, (1990).

[19] R.E.Gamboa, M.A.Muschietti and J.E.Solomin, Commun.Math.Phys. 93, 407, (1984).

[20] E.D'Hoker and D.H.Phong, Rev.Mod.Phys. 60, 917, (1988).

[21] K.Furuya, R.E.Gamboa and F.A.Schaposnik, Nucl.Phys. B208, 159, (1982).

[22] M.Kohmoto, N.den Nijs and L.P.Kadanoff, Phys.Rev.B 24, 5229, (1981).

[23] I.Enting, J. Phys. A: Math. Gen. 8, L35, (1975).

[24] L.Kadanoff, Phys.Rev.Lett. 39, 903, (1977).

[25] J.Drugowich de Felicio and R.Koberle, Phys.Rev.B 25, 511, (1982).

[26] C.Naón, J. Phys. A: Math. Gen. 22, L207, (1989).

[27] D.Friedan, E.Martinec and S.Shenker, Nucl.Phys. B271, 93, (1986).

[28] A.B.Zamolodchikov, Sov. Phys. JETP Lett.43, 731, (1986). 